\title{
Marfan syndrome affecting four generations of a family without ocular involvement
}

\author{
A.B. Bridges, M. Faed ${ }^{1}$, M. Boxer ${ }^{1}$, W.M. Haining ${ }^{2}$, T.H. Pringle and \\ G.P. McNeill
}

Departments of Cardiology, ${ }^{1}$ Human Genetics and ${ }^{2}$ Ophthalmology, Ninewells Hospital and Medical
School, University of Dundee, Dundee DDI 9SY, UK

\begin{abstract}
Summary: Thirty eight relatives of a patient with Marfan syndrome were screened for the presence of this disorder. Marfan syndrome was newly diagnosed in living members of 4 generations in this family without evidence of ocular abnormality in any. After screening, 10 relatives were newly diagnosed as having definite, and 5 relatives as having possible, Marfan syndrome. Family screening has drawbacks as well as benefits for the patients. The main benefit is the identification and treatment of previously undiagnosed patients at risk of cardiac complications which are the major cause of mortality. The drawbacks include employment problems created for patients with Marfan syndrome as a direct consequence of our screening programme and the anxiety induced in previously asymptomatic family members who did not realize that they could be at risk. Also, the 4 adult patients with possible Marfan syndrome found it difficult to accept that a definite diagnosis could not be reached after they had been invited to attend a screening programme for a serious genetic disorder.

This report illustrates the importance of screening all the relatives of a patient with Marfan syndrome to identify previously undiagnosed cases. However, before screening a family, the physician should be aware that a clear diagnosis may not be reached in all patients, and financial, psychological or social problems may arise as a result of the screening programme.
\end{abstract}

\section{Introduction}

Marfan syndrome is transmitted by an autosomal dominant gene with variable expressivity resulting in a wide range of clinical features, which may cause problems in diagnosis. The clinical features include cardiac, skeletal, ocular, pulmonary, central nervous system and dermatological involvement. The most important complications are cardiac which are the major causes of death in these patients. The diagnosis of Marfan syndrome is usually based on the criteria suggested by Pyeritz and McKusick, ${ }^{1}$ who suggested that at least two of the following criteria be fulfilled to make the diagnosis of Marfan syndrome in any one individual: characteristic family history, ocular, cardiac or skeletal features. Super ${ }^{2}$ proposed a system of counting major and minor criteria to aid in the diagnosis. The major criteria included mitral regurgitation with a floppy valve, aortic regurgitation, dissection of an aortic aneurysm, lens dislocation and a trembling iris. The minor criteria included the skeletal features, spontaneous pneumothorax,

Correspondence: A.B. Bridges, M.R.C.P.

Accepted: 10 December 1990 floppy mitral valve with no regurgitation and severe myopia. Super suggested that patients with one major and two minor criteria, five minor criteria and a positive family history of Marfan syndrome or seven minor criteria and no family history of Marfan syndrome may benefit from medical surveillance. However, it has been stated that patients with Ehlers-Danlos syndrome Type IV may be falsely diagnosed as having Marfan syndrome using Super's system. ${ }^{3}$ Due to the large clinical variability in the expression of this gene, the diagnosis of Marfan syndrome can be difficult despite the current diagnostic guidelines.

\section{Case report}

In 1984 a 39 year old man (II 4) was admitted in acute left ventricular failure secondary to a proximal ascending aortic aneurysm with severe aortic regurgitation. Examination at the time revealed the clinical stigmata of Marfan syndrome. He was transferred urgently to a cardiothoracic unit where an emergency prosthetic replacement of the aortic valve and proximal ascending aorta was performed. Following discharge he was followed up in 
the cardiology clinic where he was noted to have a small paravalvular leak and enlarging ascending aortic aneurysm. In 1988 he was again admitted as an emergency following an episode of severe chest and back pain. A computed tomographic (CT) scan showed that the aortic root was $11 \mathrm{~cm}$ in diameter and aortic arch angiography demonstrated leakage of the contrast out of the valve conduit into an aneurysm sac. The patient was taken to theatre where dehiscence of the distal graft anastomosis and coronary suture lines were found and a repair of the disrupted aortic dacron graft prosthesis was performed. The patient was later transferred back to the referring hospital for postoperative convalescence and at this time a full family history was obtained.

\section{Family investigation}

On the basis of the family details provided, 39 members of his family from 4 generations, ranging from 1 to 77 years of age, were invited by letter to attend as outpatients to be screened for Marfan syndrome. Thirty eight members of the family attended and consented to be screened, all had a clinical examination and echocardiograms performed. Each patient was then referred to the ophthalmologists and reviewed after their ocular examination. II 14 was not invited to the screening clinic as she lives abroad, III 16 was invited but did not attend.

A family pedigree (Figure 1) was constructed from information provided by the family, from case notes and from the death certificates of deceased members which were examined and assessed.

\section{Results}

Two deceased members (I 4, II 7), recognized to be at $50 \%$ risk of being affected, died from aortic aneurysm dissections which can be taken to indicate the presence of Marfan syndrome in these individuals. The death certificate of another member of the family (II 11) states she died, aged 34, from asphyxia secondary to an epileptic seizure. One of her children has Marfan syndrome indicating that the deceased patient was an obligate carrier of the gene. The diagnosis of Marfan syndrome in the living members of this family was based on the criteria suggested by Pyeritz and McKusick. ${ }^{1}$ After completion of the screening, 12 living members of this family were diagnosed as having definite Marfan syndrome. This group comprised the propositus, one of his sons, 9 newly diagnosed adults and one newly diagnosed child. The clinical features of the 11 adults with definite Marfan syndrome are given in Table I. One of the propositus' sons had been diagnosed as having Marfan syndrome but his other son had not been previously screened. The diagnosis of Marfan syndrome was definitely excluded in 22 other members of the family. Four adult patients were diagnosed as possible Marfan syndrome and their clinical features are given in Table II. The diagnosis of possible Marfan syndrome in these patients was based on the presence of a few skeletal signs only. There was no cardiac involvement. Figure 2 summarizes the results of the screening programme.

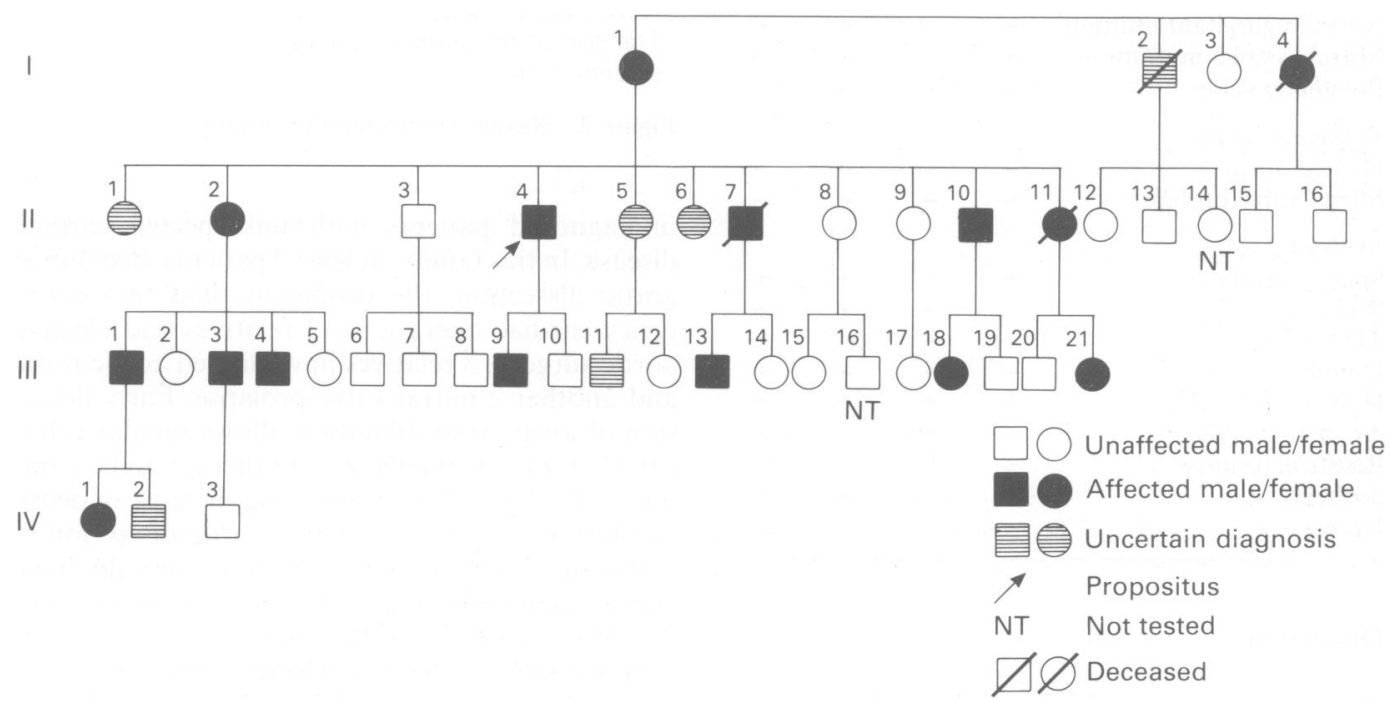

Figure 1 Family pedigree. 
Table I Clinical features of 11 adults with definite Marfan's syndrome

\begin{tabular}{|c|c|c|c|c|c|c|c|c|c|c|c|}
\hline & $I 1$ & II 2 & II 4 & II 10 & III 1 & III 3 & III 4 & III 9 & III 13 & III 18 & III 21 \\
\hline \multicolumn{12}{|l|}{ Ocular } \\
\hline Ectopia lentis & - & - & - & - & DNA & - & - & - & - & DNA & ? \\
\hline Myopia & - & - & - & - & & - & - & - & - & & - \\
\hline Retinal detachment & - & - & - & - & & - & - & - & - & & - \\
\hline \multicolumn{12}{|l|}{ Cardiac } \\
\hline Aortic regurgitant murmur & - & + & - & - & - & - & - & - & - & - & - \\
\hline Systolic murmur & - & - & - & - & - & - & - & - & + & - & + \\
\hline Prosthetic valve & + & - & + & - & - & - & - & - & - & - & - \\
\hline \multicolumn{12}{|l|}{ Echocardiogram } \\
\hline Dilated aorta & + & + & + & + & - & + & - & + & - & - & - \\
\hline Mitral valve prolapse & - & - & - & - & + & - & - & - & - & - & + \\
\hline \multicolumn{12}{|l|}{ Skeletal } \\
\hline Span $>$ height & + & + & + & + & + & + & + & + & + & + & + \\
\hline Wrist sign & + & + & - & - & + & + & + & + & + & - & + \\
\hline Thumb sign & + & - & - & - & + & + & + & + & + & + & + \\
\hline Umbilical sign & - & - & - & - & - & - & - & - & + & + & + \\
\hline High arched palate & - & + & + & + & + & + & + & + & + & + & + \\
\hline Arachnodactyly & + & - & + & + & - & + & + & + & + & + & + \\
\hline Pectus deformity & + & - & + & - & + & + & + & + & + & - & - \\
\hline Scoliosis & + & - & - & + & - & - & - & - & + & - & - \\
\hline Hernia & + & - & - & - & - & + & - & - & - & - & - \\
\hline
\end{tabular}

DNA - did not attend for examination; ? - possible early ectopia lentis.

Table II Clinical features of 4 adults with possible Marfans syndrome

\begin{tabular}{lcccc}
\hline & II & II 5 & II 6 & III $^{I I}$ \\
\hline $\begin{array}{l}\text { Ocular } \\
\text { Ectopia lentis }\end{array}$ & - & - & - & - \\
Myopia & - & - & - & - \\
Retinal detachment & - & - & - & - \\
$\begin{array}{l}\text { Cardiac } \\
\text { Aortic regurgitant murmur }\end{array}$ & - & - & - & - \\
Mitral systolic murmur & + & - & - & - \\
Prosthetic valve & - & - & - & - \\
$\begin{array}{l}\text { Echocardiogram } \\
\text { Dilated aorta }\end{array}$ & & & & \\
Mitral valve prolapse & - & - & - & - \\
Skeletal & - & - & - & - \\
Span $>$ height & & & & \\
Wrist sign & + & - & + & + \\
Thumb sign & - & - & + & + \\
Umbilical sign & + & + & - & + \\
High arched palate & - & - & - & - \\
Arachnodactyly & + & + & + & + \\
Pectus deformity & - & + & - & + \\
Scoliosis & - & - & - & - \\
Hernia & + & - & - & - \\
\hline
\end{tabular}

\section{Discussion}

The major benefit for this family from the screening programme has been the detection of previously
40 Patients invited for screening including propositus and 3 young children

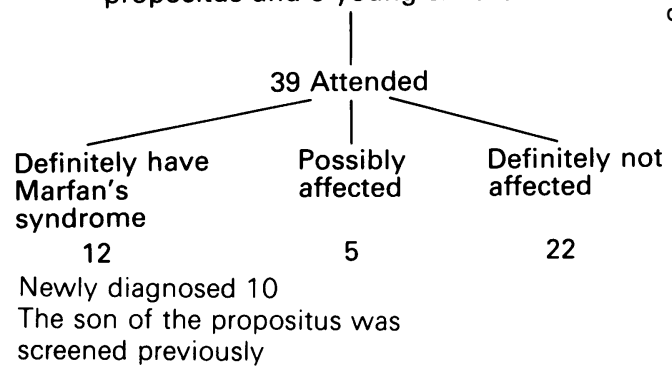

Figure 2 Result of screening programme.

undiagnosed patients with unsuspected cardiac disease. In this family, at least 2 patients died due to aortic dissection, the propositus had two emergency cardiac operations, 2 relatives had elective aortic surgery, 3 relatives have dilated aortic roots and another 2 mitral valve prolapse. Early detection of aortic root dilatation allows regular echocardiographic surveillance and therapy to be commenced to try and prevent the major cause of death in Marfan syndrome patients, aortic dissection.

Patients with Marfan syndrome should have annual echocardiograms to monitor aortic root size; if the aortic root diameter is $50 \mathrm{~mm}$ or more the echocardiogram should be repeated 3-monthly. When the aortic root diameter reaches $60 \mathrm{~mm}$ elective surgical repair is recommended as this 
prolongs life expectancy. ${ }^{4}$ The rate of aortic root dilatation is retarded by beta blockers and it is currently recommended that patients with aortic root involvement should be commenced on these drugs. Patients should also be advised to avoid strenuous or isometric exercise and contact sports. Patients with mitral or aortic valve regurgitation are at risk of infective endocarditis and require appropriate antibiotic prophylaxis. As a result of the screening programme we have been able to initiate surveillance and therapy in 7 adult patients with previously unsuspected cardiac involvement. Two patients in this group illustrate how screening asymptomatic family members of Marfan syndrome patients has both advantages and disadvantages. An asymptomatic sister of the propositus had a dilated aortic root detected at screening echocardiography which required prophylactic repair. An asymptomatic brother of the propositus lost his job directly as a result of the screening programme as he had aortic root involvement and was employed as a labourer lifting heavy weights.

This family had no individuals with certain ocular involvement; the reported incidence of lens subluxation is between $50-80 \% .^{1}$ Marfan syndrome families with no ocular manifestations have previously been reported. ${ }^{5-7}$ Ogilvie $^{5}$ described two families with no ocular involvement which had 2 and 3 affected members. Francomano ${ }^{6}$ reported that 5 out of 12 families studied had no ocular features but the numbers of affected members per family is not mentioned. Boileau ${ }^{7}$ reported a large family with 19 affected members none of whom had ocular involvement. As the incidence of ocular involvement is $50-80 \%$, the absence of ocular features in some Marfan syndrome families will occur by chance alone. However, in large families the absence of ocular features is more likely to be significant.

The lack of ocular involvement in the family described in this report is important for 2 main reasons. Firstly, it raises the possibility that this family may have a genetic mutation which does not manifest the ocular complications of Marfan syndrome. The variability of the Marfan syndrome phenotype may result from heterogeneous underlying genetic defects. Families with no ocular involvement may therefore represent one particular genetic subset of Marfan syndrome patients. Thus, the term Marfan syndrome may encompass several distinct genetic entities and therefore genetic advice to an individual should take into account the particular pattern of disease within that family and not assume Marfan syndrome is a single disease. Secondly, the lack of eye involvement throughout this family removes one of the four diagnostic criteria suggested by Pyeritz and McKusick, ${ }^{1}$ the diagnosis then depends on the presence or absence of cardiac and/or skeletal features.
As a result of screening this family, three groups of patients were identified. Those who clearly had Marfan syndrome, those who did not, and those in whom the diagnosis could not definitely be excluded. The group in whom the diagnosis was unclear were the most difficult to advise and manage. All the patients in this group knew of the morbidity and mortality of this disease; they had all consented to being screened as they felt it was in their interest to know their own diagnosis. However, after the screening we were unable to give these 4 adult patients and one child a definite diagnosis. In 3 of the cases the patients were middle aged females with normal echocardiograms and thus are unlikely to develop any serious cardiac complications of Marfan syndrome. These 3 patients are all post-menopausal and thus did not need genetic counselling. However, the fourth adult patient in this group was a 21 year old male with some of the skeletal features, a normal echocardiogram and, as with the rest of the family, no ocular signs. Marfan syndrome cannot definitely be excluded, so he will require follow-up with regular echocardiograms to detect the development of any potential cardiac complications. This patient will be reassessed at a later date in an attempt to determine if any signs have developed or changed in the hope we can be more definite about the diagnosis.

This report demonstrates the importance of screening all relatives of a patient with Marfan syndrome as this led to the discovery of 9 new adult cases and one child with Marfan syndrome. It has been possible to initiate therapy in this group in an attempt to prevent the major cardiac complication of aortic dissection. In addition to the beneficial effects of medical therapy, there are negative effects associated with the diagnosis of Marfan syndrome which may be difficult for a patient with a definite diagnosis to accept. These negative effects, which may be financial, psychological or social, should be taken into account when screening is initiated. This report also illustrates that family screening for Marfan syndrome may detect individuals in whom the diagnosis is unclear and therefore, prior to screening, patients should be informed that a clear diagnosis may not be reached. For the group with an unclear diagnosis, the uncertainty created by the screening is very confusing. The lack of ocular complications in this family is unusual and may have contributed to the difficulty of diagnosis in some cases as ocular involvement is one of the four cardinal features on which the diagnosis is usually based. The need for a reliable laboratory test to aid the diagnosis of Marfan syndrome is clear. Kainulainen et al. have recently mapped a gene causing Marfan syndrome to chromosome 15 which may be the first step in identifying the gene and hence lead to the development of a diagnostic test. ${ }^{8}$ 


\section{Acknowledgements}

We would like to thank Drs R. Wilkie and V. Marrian for

\section{References}

1. Pyeritz, R.E. \& McKusick, V.A. The Marfan syndrome: diagnosis and management. N Engl J Med 1979, 300: 772-776.

2. Super, M. Diagnosing Marfan syndrome. Br Med J 1988, 296: 1347.

3. Child, A.H. Diagnosing Marfan syndrome (letter). Br Med J 1988, 296: $1673-1674$.

4. Gott, V.L., Pyeritz, R.E., MacGovern, G.J., Cameron, D.E. \& McKusick, V.A. Surgical treatment of aneurysms of the ascending aorta in the Marfan syndrome. N Engl J Med 1986, 314: $1070-1074$.

5. Ogilvie, D.J., Wordsworth, B.P., Priestley, L.M. et al. Segregation of all four major fibrillar collagen genes in the Marfan syndrome. Am J Hum Genet 1987, 41: 1071-1082. their advice and assistance in the management of these patients.

6. Francomano, C.A., Streeten, E.A., Meyers, D.A. \& Pyeritz R.E. Marfan syndrome: exclusion of genetic linkage to the three major collagen genes. Am J Med Genet 1988, 29: 457-462.

7. Boileau, C., Jondeau, G., Bonaiti, C. et al. Linkage analysis of five fibrillar collagen loci in a large French Marfan syndrome family. J Med Genet 1990, 27: 78-81.

8. Kainulainen, K., Pulkkinen, L., Savolainen, A., Kaitila, I. \& Peltonen, L. Location on chromosome 15 of the gene defect causing Marfan syndrome. N Engl J Med 1990, 323: 935-939. 